\title{
Modeling epileptic dynamics in the hippocampus using a multiscale approach
}

\author{
Sébastien Naze*, Christophe Bernard, Viktor Jirsa \\ From Twenty Second Annual Computational Neuroscience Meeting: CNS*2013 \\ Paris, France. 13-18 July 2013
}

Epileptic seizures are characterized dynamically on multiple scales in space and time. Understanding the relations between components within and across those scales is among the fundamental goals of computational neuroscience $[1,2]$, together with the analysis of the dynamical repertoire of such a neural system $[3,4]$. Previous research in this field has developed mathematical models that can reproduce seizure dynamics as either autonomous or driven processes [5]. In this study, we start from a phenomenological model displaying features of electrical epileptic neural activity, and we move towards a more biologically realistic network of neurons, while keeping track of the dynamical repertoire exhibited by the system [6]. Our system is composed of two neuronal populations, characterized by fast excitation and slow inhibition. The first one is modeled by 3 dimensional Hindmarsh-Rose oscillators, while the other is modeled by 2-dimensional Morris-Lecar oscillators. Both pools of fast and slow dynamics do not require manual drive in order to exhibit a repertoire of regimes that are commonly encountered in epileptic brain activity. We systematically run numerical parameter space exploration at each scale before going down to a lower scale with increased complexity.

Through this framework, we investigate plausible biological causation of epileptic dynamics such as electrical and chemical synaptic connectivity, excitation/inhibition ratios, and combine them to experimental data recorded from rodent hippocampus. This multiscale architecture allows to (i) perform automatic dynamical analysis of a particular neural system, (ii) characterize the level of biological accuracy required to portray epi-phenomena, and (iii) design new experiments to comprehend seizure mechanisms. We discuss the validity of our results with regard to both biologically and computationally oriented investigations that have been performed on the topic to provide a line of thoughts for future research.

Published: 8 July 2013

\section{References}

1. Deco G, Jirsa VK, Robinson PA, Breakspear M, Friston K: "The Dynamic Brain: From Spiking Neurons to Neural Masses and Cortical Fields.". PLOS Computational Biology 2008, 4:e1000092, no. 8.

2. Rodrigues S, Chizhov AV, Marten F, Terry JR: "Mappings Between a Macroscopic Neural-mass Model and a Reduced Conductance-based Model.". Biological Cybernetics 2010, 102:361-371, no. 5.

3. Destexhe A, Contreras D, Sejnowski TJ, Steriade M: "A Model of Spindle Rhythmicity in the Isolated Thalamic Reticular Nucleus.". Journal of Neurophysiology 1994, 72:803-818, no. 2.

4. Wendling F, Bartolomei F, Bellanger JJ, Chauvel P: "Epileptic Fast Activity Can Be Explained by a Model of Impaired GABAergic Dendritic Inhibition.". European Journal of Neuroscience 2002, 15:1499-1508, no. 9.

5. Destexhe A: "Spike-and-wave Oscillations Based on the Properties of GABAB Receptors.". The Journal of Neuroscience 1998, 18:9099-9111, no. 21.

6. Stefanescu, Roxana A, Viktor KJirsa: "A Low Dimensional Description of Globally Coupled Heterogeneous Neural Networks of Excitatory and Inhibitory Neurons.". In PLoS Computational Biology Karl J. Friston 2008, 4: e1000219, no. 11.

doi:10.1186/1471-2202-14-S1-P194

Cite this article as: Naze et al.: Modeling epileptic dynamics in the hippocampus using a multiscale approach. BMC Neuroscience 201314 (Suppl 1):P194.

\footnotetext{
* Correspondence: sebastien.naze@univ-amu.fr

Institut de Neuroscience des Systèmes, UMR Inserm 1106, Aix-Marseille

Université, Faculté de Médecine, 27 Bd Jean Moulin, 13005 Marseille, France
}

( 2013 Naze et al; licensee BioMed Central Ltd. This is an Open Access article distributed under the terms of the Creative Commons Attribution License (http://creativecommons.org/licenses/by/2.0), which permits unrestricted use, distribution, and reproduction in any medium, provided the original work is properly cited. 